PROCEEDINGS OF THE

AMERICAN MATHEMATICAL SOCIETY

Volume 136, Number 11, November 2008, Pages 4045-4055

S 0002-9939(08)09508-7

Article electronically published on June 26, 2008

\title{
CHARACTERIZING INDECOMPOSABLE PLANE CONTINUA FROM THEIR COMPLEMENTS
}

\author{
CLINTON P. CURRY, JOHN C. MAYER, AND E. D. TYMCHATYN
}

(Communicated by Alexander N. Dranishnikov)

\begin{abstract}
We show that a plane continuum $X$ is indecomposable iff $X$ has a sequence $\left(U_{n}\right)_{n=1}^{\infty}$ of not necessarily distinct complementary domains satisfying the double-pass condition: for any sequence $\left(A_{n}\right)_{n=1}^{\infty}$ of open arcs, with $A_{n} \subset U_{n}$ and $\overline{A_{n}} \backslash A_{n} \subset \partial U_{n}$, there is a sequence of shadows $\left(S_{n}\right)_{n=1}^{\infty}$, where each $S_{n}$ is a shadow of $A_{n}$, such that $\lim S_{n}=X$. Such an open arc divides $U_{n}$ into disjoint subdomains $V_{n, 1}$ and $V_{n, 2}$, and a shadow (of $A_{n}$ ) is one of the sets $\partial V_{n, i} \cap \partial U$.
\end{abstract}

\section{INTRODUCTION}

In this paper, a continuum is a compact, connected, nonempty metric space. A continuum is decomposable if it can be written as the union of two of its proper subcontinua; otherwise, it is indecomposable. Let $\mathbb{C}$ denote the complex plane and let $\mathbb{C}_{\infty}$ denote the Riemann sphere $\mathbb{C} \cup\{\infty\}$. A plane domain is a subset of $\mathbb{C}_{\infty}$ which is conformally isomorphic to the open unit disk $\mathbb{D} \subset \mathbb{C}_{\infty}$ (which is to say that it is open, connected, simply connected and its boundary is a nondegenerate subcontinuum of $\mathbb{C}_{\infty}$ ). If $X$ is a continuum in $\mathbb{C}_{\infty}$, the components of $\mathbb{C}_{\infty} \backslash X$ are called complementary domains and are plane domains. If $W \subset \mathbb{C}_{\infty}$, we denote the boundary of $W$ by $\partial W$. We say that a point $x$ of a continuum is buried if it does not lie on the boundary of any complementary domain. The spherical metric on $\mathbb{C}_{\infty}$ is denoted by $d$, and $H_{d}$ denotes the Hausdorff metric on the hyperspace of subcontinua of $\mathbb{C}_{\infty}$ [18, Section 4.1].

There are several ways of recognizing intrinsically that a continuum $X$ is indecomposable. For instance, $X$ is indecomposable if and only if every proper subcontinuum of $X$ is nowhere dense in $X$ [10. Also, a continuum $X$ is indecomposable if there are points $a, b, c \in X$ such that no proper subcontinuum of $X$ contains any two of these points. In this paper, we are interested in recognizing indecomposable planar continua not by intrinsic properties, but by the relationship between the continuum and its ambient space.

Received by the editors September 4, 2007.

2000 Mathematics Subject Classification. Primary 54F15; Secondary 37F20.

Key words and phrases. Indecomposable continuum, complementary domain, Julia set, complex dynamics, buried point.

The third author was supported in part by NSERC 0GP005616. We thank the Department of Mathematics and Computer Science at Nipissing University, North Bay, Ontario, for the opportunity to work on this paper in pleasant surroundings at their annual topology workshop.

(C)2008 American Mathematical Society 
Indecomposable continua arise naturally in dynamical systems [8, 7]. However, in specific dynamical systems, it is often difficult to recognize them. In complex analytic dynamics, the Julia set of a rational map $f: \mathbb{C}_{\infty} \rightarrow \mathbb{C}_{\infty}$ is the set of unstable points under iteration of $f$ (see [15] for definitions). A long-standing question in complex analytic dynamics asks: Can the Julia set of a rational function be an indecomposable continuum? Several authors have attacked this question, among them [14, 4] for polynomials, 22] for bicritical rational maps (rational maps with exactly two critical points), and [5 for Julia sets of a class of rational functions with no buried points. In this situation, it is much easier to analyze the complement of the Julia set, called the Fatou set; this motivates our interest in studying indecomposability from the point of view of a continuum's complement.

The second-named author, with various co-authors [14, 4, 5, investigated the recognition of indecomposable continua from their complements in the case that $\partial U=X$ for some complementary domain $U$ of $X$. The tool used was prime end theory, and a characterization was obtained in the context of $X$ being a Julia set, making use of the dynamics. The characterization of indecomposable continua from their complements in the current paper primarily addresses the case that $X$ is not the boundary of any of its complementary domains (which would imply that there are infinitely many complementary domains, each having boundaries nowhere dense in $X$ ). Even better, this characterization also subsumes the first case and is entirely topological.

To state our characterization theorem, we need some definitions. These concepts are related to those which arose originally in prime end theory.

Definition 1.1. Let $U$ be a plane domain. A crosscut of $U$ is an open $\operatorname{arc} A=$ $(a, b) \subset U$ such that $\bar{A}=[a, b]$ is a closed arc which meets $\partial U$ exactly in the set $\{a, b\}$. A generalized crosscut of $U$ is an open $\operatorname{arc} A \subset U$ such that $\bar{A} \backslash A \subset \partial U$.

Notice that the notion of a generalized crosscut is strictly broader than the notion of a crosscut. It is easy to see that a generalized crosscut of a domain $U$ cuts $U$ into two nonempty disjoint subdomains $V_{1}$ and $V_{2}$ such that $U=V_{1} \cup A \cup V_{2}$.

Definition 1.2. Let $U$ be a plane domain and $A$ a generalized crosscut of $U$. We call each component of $U \backslash A$ a crosscut neighborhood. If $V$ is a crosscut neighborhood determined by a generalized crosscut $A$, we call the continuum $S=$ $\partial V \cap \partial U$ a shadow of $A$.

Thus, a generalized crosscut $A$ of a domain $U$ has exactly two crosscut neighborhoods, and consequently two shadows whose union is $\partial U$. Examples below show that one or both of these shadows can be proper subcontinua of $\partial U$ or, more surprisingly, all of $\partial U$.

The limits below are interpreted in the metric $H_{d}$.

Definition 1.3. A sequence $\left(U_{n}\right)_{n=1}^{\infty}$ of (not necessarily distinct) complementary domains of a continuum $X$ satisfies the double-pass condition if, for any sequence of generalized crosscuts $A_{n}$ of $U_{n}$, there is a sequence of shadows $\left(S_{n}\right)_{n=1}^{\infty}$ of $\left(A_{n}\right)_{n=1}^{\infty}$ such that $\lim _{n \rightarrow \infty} S_{n}=X$.

In Section 3, we prove the following theorem, which is the main theorem of this paper. 
Theorem 1.4 (Characterization Theorem). A planar continuum $X$ is indecomposable if and only if it has a sequence $\left(U_{n}\right)_{n=1}^{\infty}$ of complementary domains which satisfies the double-pass condition.

\section{PARTial AND PRIOR RESUlts}

2.1. Brief history. The first partial recognition theorem for indecomposable continua from the complement is that of Kuratowski [13].

Theorem 2.1 (Kuratowski). If a plane continuum $X$ is the common boundary of three of its complementary domains, then $X$ is either indecomposable or the union of two proper indecomposable subcontinua.

The following theorem of Rutt seems quite different and is also only applicable if $X$ is the boundary of some complementary domain.

Theorem 2.2 (Rutt, [19]). If a nondegenerate plane continuum $X$ is the boundary of a complementary domain $U$ and if there is a prime end of $U$ whose impression is $\partial U=X$, then $X$ is either indecomposable or the union of two proper indecomposable subcontinua.

Without going into detail (but see [4]), the impression of a prime end of $U$ is the intersection of the shadows of a sequence $\left(A_{n}\right)_{n=1}^{\infty}$ of crosscuts of $U$ having the property that for each $n,\left(A_{m}\right)_{m>n}$ is a pairwise closure disjoint null sequence contained in one of the crosscut neighborhoods of $A_{n}$.

The connection among the theorems above is made explicit by a technical theorem of Burgess. While the original result is stated in terms of what Burgess calls simple disks, the theorem can be equivalently stated in terms of closed balls. For $a \in \mathbb{C}_{\infty}$ and $r>0$, define the ball of radius $r$ about $a$ by

$$
B_{r}(a)=\left\{z \in \mathbb{C}_{\infty} \mid d(a, z)<r\right\} .
$$

Theorem 2.3 (Burgess, [2, Theorem 9]). Let $H$ be a closed set and $X$ a continuum in the plane. Suppose $X_{1}, X_{2}$, and $X_{3}$ are subcontinua of $X$ and $D_{1}, D_{2}$, and $D_{3}$ are pairwise disjoint closed balls with $D_{i} \cap H=\emptyset$ and $\emptyset \neq D_{i} \cap X_{i}=D_{i} \cap X$ for each $i \in\{1,2,3\}$. Then there do not exist three distinct complementary domains of $X \cup H$ such that each of them intersects each of the balls $D_{i}$.

Using this theorem, Burgess proves the following recognition theorem, which also applies when the continuum is not the union of the boundaries of its complementary domains.

Corollary 2.4 (Burgess, 2, Corollary to Theorem 9]). If the plane continuum $X$ is the limit of a sequence of distinct complementary domains of $X$, then either $X$ is indecomposable or there is only one pair of indecomposable continua whose union is $X$.

As recognition theorems, the above suffer from the weakness of their conclusion. In [4, 5, dynamical considerations rule out that the Julia set of a polynomial can be the union of two proper indecomposable subcontinua. However, this is under the hypothesis that the Julia set is the boundary of one of its complementary domains. The following definition and recognition theorem appear in [5]. Since it represents a simplification of the proof in [5], we prove Theorem 2.6 making use of Theorem 2.3, 
Definition 2.5. An antichain of crosscuts of a plane domain $U$ is a sequence $\left(H_{n}\right)_{n=1}^{\infty}$ of distinct pairwise closure disjoint crosscuts of $U$ such that, for each $m$, one crosscut neighborhood of $H_{m}$ contains all the crosscuts $\left(H_{n}\right)_{n \neq m}$.

Theorem 2.6. Let $U \subset \mathbb{C}_{\infty}$ be a plane domain. Let $z \in U$. Suppose there exists an antichain $\left(H_{n}\right)_{n=1}^{\infty}$ of crosscuts of $U$ such that $\lim _{n \rightarrow \infty} \operatorname{Sh}\left(H_{n}\right)=\partial U$, where $\operatorname{Sh}\left(H_{n}\right)$ is the shadow of the crosscut neighborhood $W_{n}$ of $U \backslash H_{n}$ which misses $z$. Then $\partial U$ is indecomposable.

Proof. For a contradiction, suppose $\partial U$ satisfies the hypotheses of the theorem but may be written as the union of proper subcontinua $X_{1}$ and $X_{2}$. By passing to a subsequence, we may assume that $\left(H_{n}\right)_{n=1}^{\infty}$ converges to a point of $\partial U$. Choose disjoint closed balls $D_{1}$ and $D_{2}$ such that

(1) $z \notin D_{i}$,

(2) $D_{i} \cap H_{n}=\emptyset$ for all $i \in\{1,2\}, n \in \mathbb{N}$,

(3) $X_{i}$ intersects the interior of $D_{i}$, and

(4) $D_{i} \cap X_{j}=\emptyset$ if $i \neq j$.

Choose three crosscuts $H_{1}, H_{2}$, and $H_{3}$ so that the component $W_{i}$ of $\mathbb{C}_{\infty} \backslash\left(\partial U \cup H_{i}\right)$ missing $z$ hits both $D_{1}$ and $D_{2}$. Notice that since the crosscuts are members of an antichain, $W_{i} \cap W_{j}=\emptyset$ for distinct $i$ and $j$ in $\{1,2,3\}$. Let $R_{1}, R_{2}$, and $R_{3}$ be arcs from $z$ to $\partial U$ disjoint (except for $z$ ) from each other and from $D_{1} \cup D_{2}$, and such that each $H_{i}$ lies in a different complementary component $U_{i}$ in $U$ of

$$
X=\partial U \cup R_{1} \cup R_{2} \cup R_{3} .
$$

Notice that $W_{i} \subset U_{i}$. Define $X_{3}=R_{1} \cup R_{2} \cup R_{3}$, and let $D_{3} \subset U$ be a closed ball about $z$ which is disjoint from $D_{1}$ and $D_{2}$. Thus, each $U_{i}$ intersects each of $D_{1}$, $D_{2}$, and $D_{3}$; this contradicts Theorem 2.3, with $H=\emptyset$ in the statement.

2.2. Necessary condition. In this section we show that for a plane continuum $X$ to be indecomposable, it is necessary that $X$ have a sequence of complementary domains whose boundaries converge to $X$. The proof requires a few additional facts and definitions.

Definition 2.7. The composant, denoted $C(p)$, of a point $p$ in a continuum $X$ is the union of all the proper subcontinua of $X$ that contain $p$.

Theorem 2.8 (10]). Let $X$ be a nondegenerate indecomposable continuum. Then the following hold:

(1) X has c pairwise disjoint composants.

(2) Each composant is dense in $X$.

(3) Each composant can be written as a countable increasing union of nowhere dense proper subcontinua of $X$, converging to $X$ in the Hausdorff metric.

Definition 2.9. A connected topological space $X$ is said to be unicoherent if, for any pair $A$ and $B$ of closed, connected subsets such that $A \cup B=X$, the intersection $A \cap B$ is connected.

Note that the plane itself, an open ball, or a closed ball in the plane are each unicoherent [20]. Recall that $B_{r}(a)$ denotes the open ball of radius $r>0$ about center $a$. 
Theorem 2.10. Let $X$ be an indecomposable plane continuum. Then there is a sequence $\left(U_{n}\right)_{n=1}^{\infty}$ of (not necessarily distinct) complementary domains of $X$ such that $\lim \partial U_{n}=X$.

Proof. This is clear if $X$ is a point, so assume $X$ is a nondegenerate indecomposable continuum. Take $p, q, r \in X$, each in a different composant of $X$. For each $n \in \mathbb{N}$, define

$$
\begin{aligned}
& Q_{n}=\text { the component of } X \backslash B_{1 / n}(p) \text { containing } q, \\
& R_{n}=\text { the component of } X \backslash B_{1 / n}(p) \text { containing } r \text {. }
\end{aligned}
$$

Notice that $\lim _{n \rightarrow \infty} Q_{n}=\lim _{n \rightarrow \infty} R_{n}=X$, by Theorem 2.8, Since $Q_{n}$ and $R_{n}$ are different components of $X \backslash B_{1 / n}(p)$, they are separated in $\mathbb{C}_{\infty} \backslash B_{1 / n}(p)$ by $\mathbb{C}_{\infty} \backslash\left(B_{1 / n}(p) \cup X\right)$. Also, $Q_{n}$ and $R_{n}$ are closed in the normal space $\mathbb{C}_{\infty} \backslash B_{1 / n}(p)$, so there is a subset $K_{n}$, closed in $\mathbb{C}_{\infty} \backslash B_{1 / n}(p)$, of $\mathbb{C}_{\infty} \backslash\left(B_{1 / n}(p) \cup X\right)$ which separates $Q_{n}$ and $R_{n}$. Since $\mathbb{C}_{\infty} \backslash B_{1 / n}(p)$ is homeomorphic to the closed unit disk in the plane, it is unicoherent, so a component $L_{n}$ of $K_{n}$ is a closed (in $\mathbb{C}_{\infty} \backslash B_{1 / n}(p)$ ) and connected subset of $\mathbb{C}_{\infty} \backslash\left(B_{1 / n}(p) \cup X\right)$ which separates $Q_{n}$ and $R_{n}$ in $\mathbb{C}_{\infty} \backslash B_{1 / n}(p)$ 20. Moreover, since $L_{n} \subset \mathbb{C}_{\infty} \backslash X$, it lies in a single complementary domain $U_{n}$ of $X$. The sequence $\left(U_{n}\right)_{n=1}^{\infty}$ formed in this way is the required sequence of complementary domains.

It is evident that $\lim _{n \rightarrow \infty} \partial U_{n} \subset X$; we aim to show that $X \subset \lim _{n \rightarrow \infty} \partial U_{n}$. Choose $\epsilon>0$, and $x \in X$. Let $N \in \mathbb{N}$ such that, for all $n \geq N$,

(1) $Q_{n} \cap B_{\epsilon}(x) \neq \emptyset$,

(2) $R_{n} \cap B_{\epsilon}(x) \neq \emptyset$, and

(3) $B_{1 / n}(p) \cap B_{\epsilon}(x)=\emptyset$.

For $n \geq N$, choose $q_{n} \in Q_{n} \cap B_{\epsilon}(x)$ and $r_{n} \in R_{n} \cap B_{\epsilon}(x)$ for $n \geq N$. The straight line segment $A_{n}$ from $q_{n}$ to $r_{n}$ is a subset of $B_{\epsilon}(x)$ and, hence, of $\mathbb{C}_{\infty} \backslash B_{1 / n}(p)$, so $A_{n}$ must meet $L_{n}$, since $L_{n}$ separates $q_{n}$ from $r_{n}$ in $\mathbb{C}_{\infty} \backslash B_{1 / n}(p)$. Since $L_{n} \subset U_{n}$, $A_{n}$ intersects $U_{n} \cap B_{\epsilon}(x)$. Since $q_{n}, r_{n}$ are not in $U_{n}$ (they lie in $X$ ), $A_{n}$ intersects $\partial U_{n}$, and $\partial U_{n} \cap B_{\epsilon}(x) \neq \emptyset$. This is true for all $n \geq N$, so $x \in \liminf _{n \rightarrow \infty} \partial U_{n} \subset$ $\lim _{n \rightarrow \infty} \partial U_{n}$. This completes the proof.

\section{THE CHARACTERIZATION THEOREM}

We saw in Subsection 2.2 that having a sequence of complementary domains whose boundaries converge to $X$ is a necessary condition for the plane continuum $X$ to be indecomposable. Example 3.2 below shows that this condition is not sufficient, even if the domains are distinct, and suggests that we must find a way to rule out that the sequence of complementary domains "splits" into "halves", each of which converge to proper indecomposable subcontinua.

\subsection{Examples.}

Example 3.1. The Knaster buckethandle continuum, depicted on the left in Figure 1, is a standard example of an indecomposable continuum. It can be viewed as a disk from which successively deeper and denser fjords are dug. Notice that for any generalized crosscut drawn in its single complementary domain, infinitely many fjords lie in one crosscut neighborhood or the other, so one shadow is dense. Notice that one composant of $X$ is the one-to-one continuous image of the half-line $[0, \infty)$. The point of $X$ corresponding to the point 0 of $[0, \infty)$ is called the endpoint of $X$. For a more precise construction, see [13, Vol. II, p. 205]. 


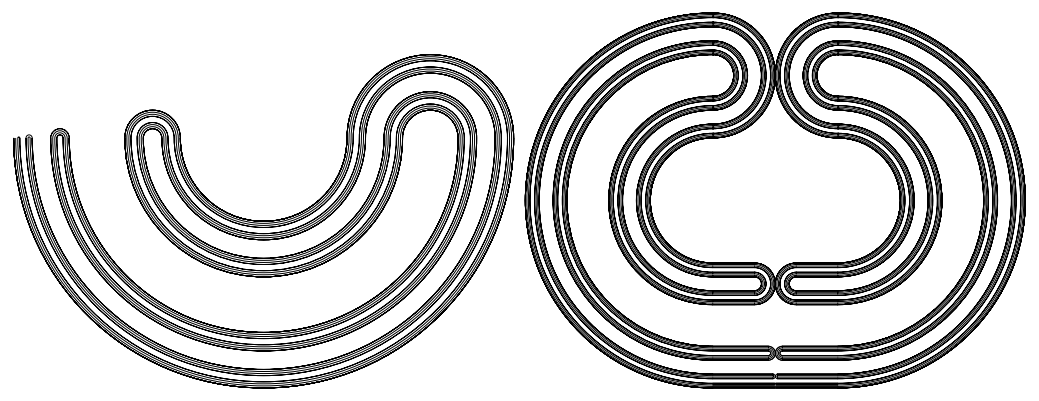

Figure 1. The Knaster buckethandle continuum (left); a union of two Knaster continua (right) meeting at a sequence of points converging to their common endpoint.

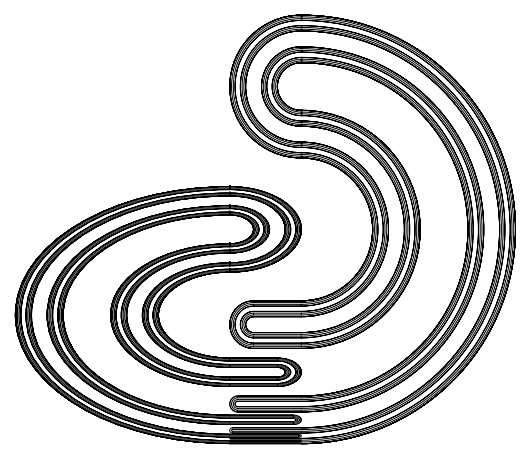

Figure 2. Two Knaster continua meeting on an end interval of each.

Example 3.2. The continuum $X$ depicted on the right in Figure 1 is an example of a continuum which satisfies the hypotheses of Theorem 2.4 without being indecomposable. It is a symmetric union of two Knaster buckethandle continua $X_{1}$ and $X_{2}$ intersecting in a countable set which lies on a vertical line. The continuum has infinitely many complementary domains $\left(U_{i}\right)_{i=1}^{\infty}$. The decomposability of $X$ can be detected from the complementary domains as follows: to its complementary domains $\left(U_{i}\right)_{i=1}^{\infty}$, associate the collection of crosscuts $\left(K_{i}\right)_{i=1}^{\infty}$, where $K_{i}$ lies in $U_{i}$ on the vertical axis of symmetry. Each crosscut has one shadow which is a subcontinuum of $X_{1}$ and another which is a subcontinuum of $X_{2}$. Therefore, any convergent sequence of shadows must converge in the limit to a proper subcontinuum of $X$.

Example 3.3. The continuum in Figure 2 is the union of a pair of Knaster continua $X_{1}$ and $X_{2}$ with distinct endpoints such that $X_{1} \cap X_{2}$ is the horizontal arc $A$ between the endpoints of $X_{1}$ and $X_{2}$. This continuum, like Example 3.1, has the property that every crosscut has a dense shadow, despite the continuum's decomposability. Let $K$ be a generalized crosscut such that one end lands on a point of $X \backslash A$ and the other end wiggles between the Knaster continua and compactifies on $A$. Neither shadow of this generalized crosscut is dense, so the constant sequence consisting of this crosscut fails the double pass condition. 
3.2. Proof of characterization theorem. In Definition 1.3. we defined the double-pass condition on a sequence of generalized crosscuts in a sequence of complementary domains which is motivated by Example 3.2 and by the similarly functioning condition of Cook and Ingram [6] introduced for recognizing indecomposable (chainable) continua in terms of refining open covers. Here we prove our main theorem: the existence of a sequence of complementary domains satisfying our double-pass condition is equivalent to indecomposability.

The following lemma follows from [23, (A1.4)], but we include a self-contained proof here for convenience.

Lemma 3.4. Suppose that $\phi: U \rightarrow \mathbb{D}$ is a conformal isomorphism, where $U$ is a plane domain. Then the image of a null sequence $\left(K_{n}\right)_{n=1}^{\infty}$ of crosscuts of $U$ is a null sequence of crosscuts of $\mathbb{D}$.

Proof. By way of contradiction, let $\left(K_{n}\right)_{n=1}^{\infty}$ be a null sequence of crosscuts of $U$ such that the image sequence $\left(A_{n}\right)_{n=1}^{\infty}=\left(\phi\left(K_{n}\right)\right)_{n=1}^{\infty}$ consists of crosscuts whose diameters are bounded away from zero. Then, by passing to a subsequence, we may assume that the image sequence accumulates on a nondegenerate continuum $L \subset \overline{\mathbb{D}}$. Since $\left(K_{n}\right)_{n=1}^{\infty}$ does not accumulate on a subset of $U$, we see that $L \subset \partial \mathbb{D}$. Also, we may assume without loss of generality that $\left(K_{n}\right)_{n=1}^{\infty}$ converges to a point of $x \in \partial U$.

Let $t \in L$. There exists a chain of crosscuts $\left(A_{n}^{\prime}\right)_{n=1}^{\infty}$ of $\mathbb{D}$ converging to $t$ which maps to a null sequence $\left(K_{n}^{\prime}\right)_{n=1}^{\infty}$ of crosscuts of $U$ by $\phi^{-1}$ (see [15, Lemma 17.9]). We may assume that the sequence $\left(K_{n}^{\prime}\right)_{n=1}^{\infty}$ converges to a point of $\partial U$ by passing to a subsequence. Since $\left(A_{n}\right)_{n=1}^{\infty}$ accumulate on $t$, all but finitely many $A_{n}$ intersect the crosscut neighborhood of $A_{m}^{\prime}$ corresponding to $t$. Also, since $\left(K_{n}\right)_{n=1}^{\infty}$ forms a null sequence in $U$, we see that all but finitely many $K_{n}$ (thus $A_{n}$ ) lie entirely within the crosscut neighborhood of $K_{m}^{\prime}$ (thus $A_{m}^{\prime}$ ) corresponding to $t$. However, the crosscut neighborhoods of $A_{m}^{\prime}$ form a null sequence, so $\left(A_{n}\right)_{n=1}^{\infty}$ form a null sequence.

We say that a pair of subsets $E_{1}$ and $E_{2}$ of $\partial \mathbb{D}$ are unlinked if there exist intervals $I_{1} \supset E_{1}$ and $I_{2} \supset E_{2}$ such that $\left|I_{1} \cap I_{2}\right| \leq 2$.

Lemma 3.5. Suppose $U \subset \mathbb{C}_{\infty}$ is a plane domain, and let $\phi: U \rightarrow \mathbb{D}$ be a conformal isomorphism. Let $B_{1}$ and $B_{2}$ be disjoint closed balls meeting $\partial U$ in their interiors, and let $E_{i} \subset \partial \mathbb{D}$ denote the endpoints of all of the crosscuts of $\mathbb{D}$ which constitute $\phi\left(\left(\partial B_{i}\right) \cap U\right)$. If $E_{1}$ and $E_{2}$ are unlinked, then there is a generalized crosscut $K$ of $U$ which separates $B_{1} \cap U$ from $B_{2} \cap U$ in $U$. Moreover, if $\partial U$ is locally connected, $K$ is a crosscut of $U$.

Proof. Let $I_{1}$ and $I_{2}$ be minimal closed intervals of $\partial \mathbb{D}$ such that $I_{i} \supset E_{i}$ for $i \in\{1,2\}$ and $\left|I_{1} \cap I_{2}\right| \leq 2$. Note that $\left(\partial B_{i}\right) \cap U$ is the union of a null sequence of crosscuts of $U$, so $\phi\left(\left(\partial B_{i}\right) \cap U\right)$ is the union of a null sequence of crosscuts of $\mathbb{D}$ by Lemma 3.4. $I_{1}$ and $I_{2}$ being unlinked implies that neither $\phi\left(B_{1} \cap U\right)$ nor $\phi\left(B_{2} \cap U\right)$ separates the other in $\mathbb{D}$. By connectedness of $\mathbb{D}$, there is then a unique component $D$ of $\mathbb{D} \backslash \phi\left(\left(B_{1} \cup B_{2}\right) \cap U\right)$ which meets both $\phi\left(\partial B_{1} \cap U\right)$ and $\phi\left(\partial B_{2} \cap U\right)$. The crosscuts on $\partial D$ form a null sequence, so it is not difficult to show that $\bar{D} \subset \overline{\mathbb{D}}$ is locally connected.

The endpoints of $I_{1}$ and $I_{2}$ are each on $\partial D$. Let $K$ be a crosscut joining an endpoint of $I_{1}$ to an endpoint of $I_{2}$ such that $K$ separates the interiors of $I_{1}$ and 
$I_{2}$, in $\partial \mathbb{D}$, and thus $\phi\left(B_{1}\right)$ and $\phi\left(B_{2}\right)$ in $\mathbb{D}$. Then $\phi^{-1}(K)$ is a generalized crosscut of $U$ which separates $B_{1} \cap U$ from $B_{2} \cap U$. Further, if $\partial U$ is locally connected, $\phi^{-1}$ extends to a continuous function $\bar{\phi}^{-1}: \overline{\mathbb{D}} \rightarrow \bar{U}$ [15, Theorem 17.14]. In this case, $\phi^{-1}(K)$ is a true crosscut of $U$, as $\overline{\phi^{-1}(K)}=\bar{\phi}^{-1}(\bar{K})$ is an arc.

Now we have the tools to prove our Characterization Theorem 1.4.

Proof of Theorem 1.4. First, suppose that $X$ is indecomposable. We show $X$ satisfies the double-pass condition. By Theorem 2.10, there exists a sequence $\left(U_{n}\right)_{n=1}^{\infty}$ of complementary domains of $X$ such that $\lim _{n \rightarrow \infty} \partial U_{n}=X$. Let $\left(K_{n}\right)_{n=1}^{\infty}$ be a sequence of generalized crosscuts, with $K_{n}$ in $U_{n}$ for each $n \in \mathbb{N}$. Let $A_{n}$ and $B_{n}$ be the shadows of $K_{n}$, with $H_{d}\left(A_{n}, X\right) \leq H_{d}\left(B_{n}, X\right)$, where $H_{d}$ denotes the Hausdorff metric.

We claim that $\lim _{n \rightarrow \infty} A_{n}=X$. Since the hyperspace of subcontinua of $X$ is a compact metric space, it is sufficient to show that every convergent subsequence of $\left(A_{n}\right)_{n=1}^{\infty}$ converges to $X$. Let $\left(n_{i}\right)_{i=1}^{\infty}$ be such that $\left(A_{n_{i}}\right)_{i=1}^{\infty}$ converges to a continuum $A \subset X$. By passing to a subsequence, we may assume $\left(B_{n_{i}}\right)_{i=1}^{\infty}$ also converges to a continuum $B \subset X$. Since $\lim _{i \rightarrow \infty} \partial U_{n_{i}}=X$ and $A_{n_{i}} \cup B_{n_{i}}=\partial U_{n_{i}}$, we have that $A \cup B=X$. Since $X$ is indecomposable, $A$ and $B$ may not both be proper subcontinua of $X$, so $A=X$ or $B=X$. Since $H_{d}\left(A_{n_{i}}, X\right) \leq H_{d}\left(B_{n_{i}}, X\right)$ for all $i$, we have $A=X$. This concludes the proof of this implication.

Now we prove the converse. Let $X$ be a continuum with a sequence $\left(U_{n}\right)_{n=1}^{\infty}$ of complementary domains satisfying the double-pass condition. Suppose, by way of contradiction, that $X=X_{1} \cup X_{2}$, where $X_{1}$ and $X_{2}$ are proper subcontinua of $X$. We can then find open balls $B_{1}$ and $B_{2}$ such that

(1) $\bar{B}_{1} \cap \bar{B}_{2}=\emptyset$,

(2) $B_{i} \cap X_{i} \neq \emptyset$ for $i=1,2$, and

(3) $\bar{B}_{i} \cap X_{j}=\emptyset$ for $i \neq j$.

Since $\left(U_{n}\right)_{n=1}^{\infty}$ satisfies the double-pass condition, there exists a particular $N \in \mathbb{N}$ such that, for any generalized crosscut $K$ of $U_{N}$, one shadow of $K$ intersects both $B_{1}$ and $B_{2}$. We fix $U=U_{N}$, and let $\phi: U \rightarrow \mathbb{D}$ be a conformal isomorphism. Define $E_{1}$ and $E_{2}$ as in Lemma 3.5 to be the sets of endpoints of the crosscuts comprising $\phi\left(\left(\partial B_{1}\right) \cap U\right)$ and $\phi\left(\left(\partial B_{2}\right) \cap U\right)$, respectively. There are two cases: Either $E_{1}$ and $E_{2}$ are linked or they are not. The second case cannot occur, as Lemma 3.5 asserts the existence of a generalized crosscut $K_{0}$ of $U$ separating $B_{1} \cap U$ and $B_{2} \cap U$, contrary to our assumption.

Thus, $E_{1}$ and $E_{2}$ are linked. Note that each of $\phi\left(\left(\partial B_{1}\right) \cap U\right)$ and $\phi\left(\left(\partial B_{2}\right) \cap U\right)$ consists of crosscuts of $\mathbb{D}$ with endpoints in $E_{1}$ and $E_{2}$, respectively. There are two cases: (1) either one of $\phi\left(\left(\partial B_{1}\right) \cap U\right)$ or $\phi\left(\left(\partial B_{2}\right) \cap U\right)$ separates the other in $\mathbb{D}$ or $(2)$ neither $\phi\left(\left(\partial B_{1}\right) \cap U\right)$ nor $\phi\left(\left(\partial B_{2}\right) \cap U\right)$ separates the other in $\mathbb{D}$. In each case, we construct a crosscut $A \subset \mathbb{D}$. This crosscut will have the property that $\phi^{-1}(A)$ is a crosscut of $U$, which we show leads to a separation of one of $X_{1}$ or $X_{2}$, a contradiction.

In case (1), without loss of generality, $\phi\left(\left(\partial B_{1}\right) \cap U\right)$ separates $\phi\left(\left(\partial B_{2}\right) \cap U\right)$ in $\mathbb{D}$. Since $\mathbb{D}$ is unicoherent, a component of $\phi\left(\left(\partial B_{1}\right) \cap U\right)$ also separates $\phi\left(\left(\partial B_{2}\right) \cap U\right)$, so a crosscut in $\phi\left(\left(\partial B_{1}\right) \cap U\right)$ does. Let $A$ be this crosscut. Then $\phi^{-1}(A)$ is a crosscut of $U$ separating $B_{2} \cap U$ in $U$.

For case (2), we suppose that neither $\phi\left(\left(\partial B_{1}\right) \cap U\right)$ nor $\phi\left(\left(\partial B_{2}\right) \cap U\right)$ separates the other in $\mathbb{D}$. Since $E_{1}$ and $E_{2}$ are linked, let $e_{1}$ and $e_{1}^{\prime}$ be points of $E_{1}$ separated 
in $\partial \mathbb{D}$ by points of $E_{2}$. Let $K_{1}$ and $K_{1}^{\prime}$ be crosscuts of $\mathbb{D}$ in $\phi\left(\left(\partial B_{1}\right) \cap U\right)$ that have $e_{1}$ and $e_{1}^{\prime}$, respectively, as endpoints. Since $\phi\left(\left(\partial B_{2}\right) \cap U\right)$ does not separate $K_{1}$ from $K_{1}^{\prime}$ in $\mathbb{D}$, there is an arc $C$ from a point of $K_{1}$ to a point of $K_{1}^{\prime}$ in $\mathbb{D} \backslash \phi\left(\bar{B}_{2} \cap U\right)$. Let $A \subset K_{1} \cup K_{1}^{\prime} \cup C$ be a crosscut of $\mathbb{D}$ from $e_{1}$ to $e_{1}^{\prime}$ which then separates $\phi\left(B_{2} \cap U\right)$ in $\mathbb{D}$. Because $\phi^{-1}\left(K_{1}\right)$ and $\phi^{-1}\left(K_{1}^{\prime}\right)$ are crosscuts of $U$, we see that $\phi^{-1}(A)$ is a crosscut of $U$. Moreover, $\phi^{-1}(A)$ separates $B_{2} \cap U$ in $U$.

The proofs in cases (1) and (2) now proceed together. Let $S_{1}$ be an irreducible arc which joins points of $\phi\left(\left(\partial B_{2}\right) \cap U\right)$ which are separated by $A$; we may stipulate that $S_{1}$ intersects $A$ exactly once, transversely. By applying $\phi^{-1}$ to both $A$ and $S_{1}$, we obtain a crosscut $A^{\prime}$ of $U$ and a compact arc $S_{1}^{\prime} \subset U$ between points of $\partial B_{2}$ which intersects $A^{\prime}$ once transversely. Let $S_{2}^{\prime}$ be a compact arc in $\bar{B}_{2}$ which joins the endpoints of $S_{1}^{\prime}$. Then $S_{1}^{\prime} \cup S_{2}^{\prime}=S$ is a simple closed curve. Observe that $S \cap X_{1}=\emptyset$, since $S_{1}^{\prime} \subset U$ and $S_{2}^{\prime} \subset \bar{B}_{2}$. However, the compact arc $\overline{A^{\prime}}$ joins points of $X_{1}$ and intersects $S$ exactly once, transversely. Thus, some point of $X_{1}$ lies inside and another point lies outside of $S$, while $X_{1} \cap S=\emptyset$, contradicting the connectedness of $X_{1}$.

Examination of the proof of Theorem 1.4 gives a stronger theorem for continua whose complementary domains have locally connected boundaries.

Definition 3.6. A sequence of complementary domains $\left(U_{n}\right)_{n=1}^{\infty}$ satisfies the crosscut condition if, for every sequence of crosscuts $\left(A_{n}\right)_{n=1}^{\infty}, A_{n} \subset U_{n}$, there exists a choice of shadows $S_{n}$ of $A_{n}$ such that $\lim S_{n}=X$.

Example 3.3 showed that this is a strictly weaker condition than the double-pass condition. However, the following shows that for a certain class of continua, the two notions are equivalent.

Corollary 3.7. A planar continuum $X$ whose complementary domains have locally connected boundaries is indecomposable if and only if it has a sequence $\left(U_{n}\right)_{n=1}^{\infty}$ of complementary domains which satisfies the crosscut condition.

This follows from the proof of Theorem 1.4 since the generalized crosscut of $U$ constructed in the proof with Lemma 3.5 is a crosscut if $\partial U$ is locally connected.

\section{Questions And Further Results}

We close with a question about rational Julia sets for which our Characterization Theorem may prove useful and two theorems by the first author that will appear in a subsequent paper extending our results to surfaces.

Question 4.1. Let $J=J(R)$ be the Julia set of a rational function $R: \mathbb{C}_{\infty} \rightarrow$ $\mathbb{C}_{\infty}$ and suppose that $J$ has buried points. Can $J$ be the union of two proper indecomposable subcontinua? In particular, can $J$ contain a proper indecomposable subcontinuum with interior in $J$ ?

Definition 4.2. A surface is a connected Hausdorff space with a countable basis, each point of which has a neighborhood homeomorphic to an open ball in the plane. Let $X$ be a continuum in the surface $S$. As before, a component of $S \backslash X$ is called a complementary domain.

Definition 4.3. A connected topological space $X$ is multicoherent of degree $k$ if, for any pair of closed, connected sets $A$ and $B$ such that $A \cup B=X$, the intersection $A \cap B$ consists of at most $k$ components. 
A complementary domain in a surface, unlike in the planar case, need not be simply connected. Using the notion of multicoherence and its consequences (see 21. Theorem 1] for the relevant extension of the Phragmén-Brouwer theorem), we can prove the following theorem. We omit the proof, which is similar to the proof of Theorem 2.10.

Theorem 4.4. Let $S$ be a compact surface and $X$ an indecomposable subcontinuum of $S$. Then there is a sequence $\left(U_{n}\right)_{n=1}^{\infty}$ of complementary domains of $X$ such that $\lim \partial U_{n}=X$.

We claim in Theorem 4.4 that having a sequence of complementary domains converging to $X$ is a necessary condition for a continuum $X$ contained in a surface $S$ to be indecomposable. We saw in the plane a partial converse: given a sequence of distinct complementary domains $\left(U_{n}\right)_{n=1}^{\infty}$ such that $\lim \partial U_{n}=X$, it follows that $X$ is either indecomposable or the union of two proper indecomposable subcontinua (Theorem 2.4). In this connection, we close with the following two theorems generalizing Burgess's Theorem 2.4 and our Characterization Theorem 1.4 to continua in surfaces, proofs of which will appear subsequently in a paper by the first-named author.

Theorem 4.5. Let $S$ be a compact surface. Suppose a continuum $X \subset S$ has a sequence $\left(U_{n}\right)_{n=1}^{\infty}$ of distinct complementary domains with $\lim \partial U_{n}=X$. Then either $X$ is indecomposable or there is only one pair of indecomposable subcontinua whose union is $X$.

Theorem 4.6. Let $S$ be a compact surface. Suppose the continuum $X \subset S$ has buried points. Then $X$ is indecomposable iff $X$ has a sequence $\left(U_{n}\right)_{n=1}^{\infty}$ of distinct complementary domains satisfying the double-pass condition: for any sequence $\left(A_{n}\right)_{n=1}^{\infty}$ of generalized crosscuts (suitably defined), with $A_{n} \subset U_{n}$, there is a sequence of shadows $\left(S_{n}\right)_{n=1}^{\infty}$, where each $S_{n}$ is a shadow of $A_{n}$, such that $\lim S_{n}=X$.

\section{REFERENCES}

[1] P. Blanchard, R. L. Devaney, D. M. Look, P. Seal, and Y. Shapiro. Sierpinski-curve Julia sets and singular perturbations of complex polynomials. Ergodic Theory and Dynamical Systems. 25:1047-1055, 2005. MR.2158396 (2006d:37087)

[2] C. E. Burgess. Continua and their complementary domains in the plane. Duke Math Journal. 18:901-917, 1951. MR0044828(13:484d)

[3] L. Carleson and T. W. Gamelin. Complex Dynamics. Springer-Verlag, New York, Berlin, 1993. MR.1230383 (94h:30033)

[4] D. K. Childers, J. C. Mayer, and J. T. Rogers, Jr. Indecomposable continua and the Julia sets of polynomials, II. Topology and Its Applications. 153:1593-1602, 2006. MR2216123 (2007c:37058)

[5] D. K. Childers, J. C. Mayer, H. M. Tuncali, and E. D. Tymchatyn. Indecomposable continua and the Julia sets of rational maps. Complex dynamics. Contemporary Mathematics. 396:120, Amer. Math. Soc., Providence, RI, 2006. MR2209083 (2007a:37060)

[6] Howard Cook and W. T. Ingram. A characterization of indecomposable compact continua. Topology Conference (Arizona State Univ., Tempe, Ariz., 1967). 168-169, Arizona State Univ., Tempe, AZ, 1968. MR0253298 (40:6513)

[7] R. L. Devaney and X. Jarque. Indecomposable continua in exponential dynamics. Conform. Geom. Dyn. 6:1-12, 2002. MR1882085 (2003e:37060)

[8] R. L. Devaney. Cantor and Sierpinski, Julia and Fatou: Complex topology meets complex dynamics. Notices of the AMS. 51:9-15, 2004. MR2022671 (2004i:37092)

[9] T. W. Gamelin. Complex Analysis. Springer-Verlag, New York, 2000. MR1830078 (2002h:30001) 
[10] J. Hocking and G. Young. Topology. Addison-Wesley, Reading, MA, 1961. MR0125557 (23:A2857)

[11] J. Krasinkiewicz. On homeomorphisms of the Sierpiński curve. Prace Mat. 12:255-257, 1969. MR0247618(40:882)

[12] J. Krasinkiewicz. On internal composants of indecomposable plane continua. Fund. Math. 84:255-263, 1974. MR0339101 (49:3864)

[13] K. Kuratowski. Topology, Volume II. Academic Press, New York, 1968. MR0259835(41:4467)

[14] J. C. Mayer, and J. T. Rogers, Jr. Indecomposable continua and the Julia sets of polynomials. Proc. AMS. 117:795-802, 1993. MR.1145423 (93d:58138)

[15] J. Milnor. Dynamics in One Complex Variable: Introductory Lectures, 2nd Edition. Vieweg, Braunschweig/Weisbaden, 2000. MR1721240 (2002i:37057)

[16] J. Milnor. On rational maps with two critical points. Experimental Math. 9:481-522, 2000. MR1806289 (2001k:37074)

[17] J. Milnor. Geometry and dynamics of quadratic rational maps. Experimental Math. 2(1): 37-83, 1993. MR1246482 (96b:58094)

[18] S. B. Nadler. Continuum Theory. Marcel Dekker, New York, 1992. MR.1192552 (93m:54002)

[19] N. E. Rutt. Prime ends and indecomposability. Bull. AMS. 41:265-273, 1935.

[20] A. H. Stone. Incidence relations in unicoherent spaces. Trans. Amer. Math. Soc. 65:427-447, 1949. MR0030743 (11:44g)

[21] A. H. Stone. Incidence relations in multicoherent spaces. I. Trans. Amer. Math. Soc. 66:389406, 1949. MR0030744(11:45a)

[22] Y. Sun and C-C. Yang. Buried points and Lakes of Wada continua. Discrete and Continuous Dynamical Systems. 9(2):379-382, 2003. MR1952380(2003m:37064)

[23] H. D. Ursell and L. C. Young. Remarks on the theory of prime ends. Memoirs of the American Mathematical Society. 3, 1951. MR0042110(13:55a)

Department of Mathematics, University of Alabama at Birmingham, Birmingham, Alabama 35294-1170

E-mail address: clintonc@uab.edu

Department of Mathematics, University of Alabama at Birmingham, Birmingham, Alabama 35294-1170

E-mail address: mayer@math.uab.edu

Department of Mathematics and Statistics, University of Saskatchewan, Saskatoon, Saskatchewan, Canada S7N 0W0

E-mail address: tymchat@math.usask.ca 